\title{
Grid Voltage Harmonic Damping Method for SPC based Power Converters with Multiple Virtual Admittance Control
}

\author{
Andres Tarrasó, Jose Ignacio Candela, Joan Rocabert \\ Technical University of Catalonia (UPC) \\ Research Center on Renewable Electrical Energy Systems \\ (SEER Center), Terrassa, SPAIN \\ andres.tarraso@upc.edu
}

\author{
Pedro Rodriguez \\ Universidad de Loyola \\ Sevilla, SPAIN \\ prodriguez@uloyola.es
}

\begin{abstract}
The appearance of harmonics in the grid voltage is an issue for the generation systems and grid connected consumers. This paper presents the basic control strategy to be implemented in Synchronous Power Control (SPC) based power converters, which contributes to reduce the harmonic content in the voltage by means of injecting different harmonic current to the grid using a separated virtual admittance algorithm. This control strategy is capable of generating such a current reference that attenuates the harmonic values at the point of connection of the converter.
\end{abstract}

Keywords-Power quality; Power converter; Renewable enegy system; Synchronous power;

\section{INTRODUCTION}

In the last years new concepts for controlling grid-connected converters, based on importing the advantages in the performance of synchronous generators, have been developed. However, the approaches followed are different giving rise to the emulation of enhanced synchronous generators, as the one in [1], or the mimic emulation of synchronous generators [2], among others. This new concept has been integrated in different applications to improve the grid integration of renewable generation based systems, which are normally interfaced with the grid through power converters. The main principle behind this concept is to control the power injected to the grid without using any synchronization loop [3], but the power balance itself, introducing in the process terms for emulating the inertia and setting the connection admittance, so-called virtual admittance. Different authors applied different current control strategies related with the virtual impedance concept [4] in order to control grid-feeding power converters taking advantage of the synchronous generator performance in steady-state, transient or faulty conditions. The major drawback behind the virtual impedance concept is that it introduces a derivative term in the control loop. This term is affected by the measurement noise coming from the current sensors, as the derivative block reacts to these spikes, damaging thus the control performance. This problem is worse in case of harmonics where the noise presence is still higher and the control signals themselves have a higher frequency.

This paper deals with the implementation of parallel virtual admittances at harmonic frequencies. The virtual admittance approach provides advantages if compared with virtual impedance, as it does not introduce any derivative term in the controller. As the admittance is a virtual element this work takes advantage of this and considers a synchronous generation systems with different admittances at different frequencies. By means of this approach once the grid harmonic distortion is being analyzed, several harmonic virtual admittance loops can be implemented in the control of DC-AC converters, in order to attenuate the grid voltage distortion. These virtual admittances operate in parallel with the main voltage loop at fundamental frequency, in order to inject the required harmonic content at a specific frequency to enhance the voltage profile.

On this paper the scope will be focused just in the harmonic attenuation. As it will be proven, being able to inject harmonics to the grid by this synchronous power control makes it possible to inject active and reactive power and also attenuate harmonic distortion appearing at the point of common coupling (PCC).

\section{FUNDAMENTAL AND HARMONIC CURRENT CONTROL LOOP}

In this chapter the control strategy applied is based on the enhanced emulation of a synchronous generator where the synchronization loop is inherently implemented in the control equations [5]. The main mechanical characteristics of the synchronous generators, collected by the swing equations, are the mechanical inertia and the damping effect. Following the electrical characteristic of the synchronous generator is enclosed in the virtual admittance block, which is described in detail in [6].These blocks are depicted in Fig. 1. 


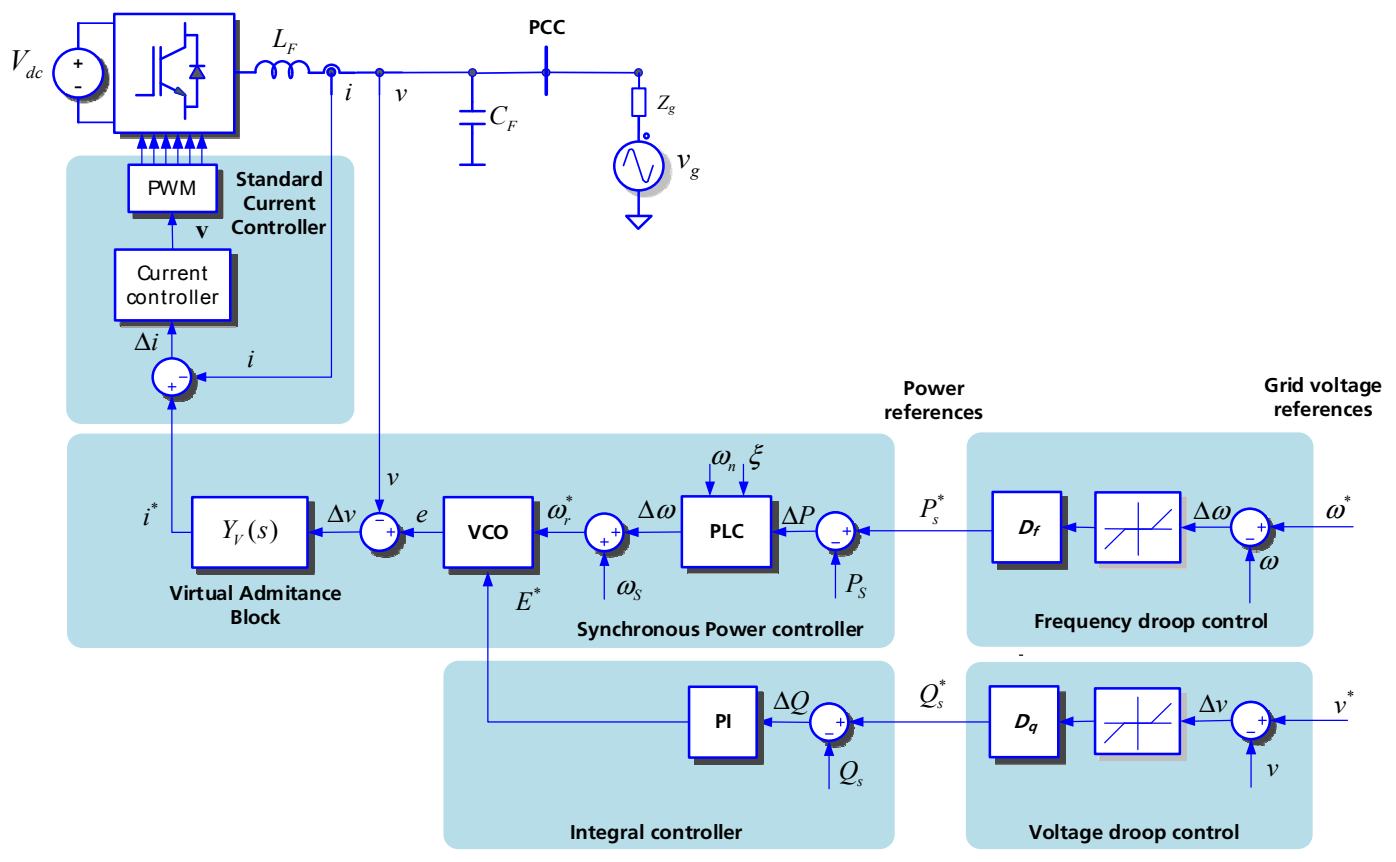

Fig. 1 Synchronous Power Converter block diagram description

An external P- $f$ and Q- $V$ droop blocks [7] are implemented in order to add supporting functionalities in the converter in case of island or weak grids scenarios, where high power injection could modify the voltage amplitude or the grid frequency [8]. Then the electromechanical equations are implemented in the Power-Loop Controller (PLC) block. Inside this block the swing equation of a synchronous generator is implemented. The main functionality of this block is to provide a virtual synchronous speed $\omega$ in order to regulate the power injected to the grid. This $\omega$ is integrated in order to obtain the virtual phase angle $\theta_{V}$ [9] and derivate $e(t)$, composed by three reference sinusoidal waveforms with phase angle, $\theta_{V}$, and amplitude, $\mathrm{E}^{*}$.

This voltage $e(t)$ is the output voltage reference, the comparison of this magnitude with the voltage measurement at the connection point of the converter, $v$, is the input signal of the electrical characteristic block in the SPC controller. Usually it is described as one admittance element composed by a virtual resistance and a virtual inductance, the transfer function is shown in the following equation (1):

$$
Y_{V}(s)=\frac{1}{R_{V}+s L_{V}}
$$

This virtual admittance can be applied not only for the fundamental frequency but also for any harmonic component existing on the grid. Considering $e(t)$ as a fundamental $50 \mathrm{~Hz}$ component, the error appearing for different harmonic components are the values existing from the voltaetru56uge measurement in the connection point of the converter $v$.
In Fig. 2 it can be seen in detail how the control strategy of the converter is able to detect the voltage distortion of the different harmonics on the grid. In the figure, four strings have been selected; each one tuned at its harmonic frequency, including the harmonic positive or negative sequence. Each horizontal branch is composed by a band-pass filter tuned at the harmonic frequency component, $h$, and the virtual admittance controlling the injection of current of either fundamental or harmonic frequency.

$$
\begin{gathered}
G_{B P}(s)=\frac{k \cdot\left(h \cdot \omega_{0}\right) \cdot s}{s^{2}+k \cdot\left(h \cdot \omega_{0}\right) \cdot s+\left(h \cdot \omega_{0}\right)^{2}} \\
Y_{V-h}(s)=\frac{1}{R_{V-h}+s \cdot L_{V-h}}
\end{gathered}
$$

In addition, to ensure the stability of operation of the converter in presence of grid harmonics, it is required to add a transient branch which will be controlling the fast dynamics and the grid perturbations for the rest of voltage components.

The expression in equation (3) provides the current reference for each voltage harmonic, which is controlled directly by the virtual admittance and its relationship between $L_{V}$ and $R_{V}$. The virtual admittance is composed by an inductor and a resistor and, as a result, a small delay on the current reference is generated. On the $50 \mathrm{~Hz}$ this additional delay on the current reference does not affect the control algorithm. However when the frequency is increased the delay increments its influence on the controller, modifying the reference and changing the controller performance and the whole harmonic compensation loop. Because of this a reduction of the $\mathrm{L} / \mathrm{R}$ ratio is needed when trying to control harmonics above the nominal frequency. 


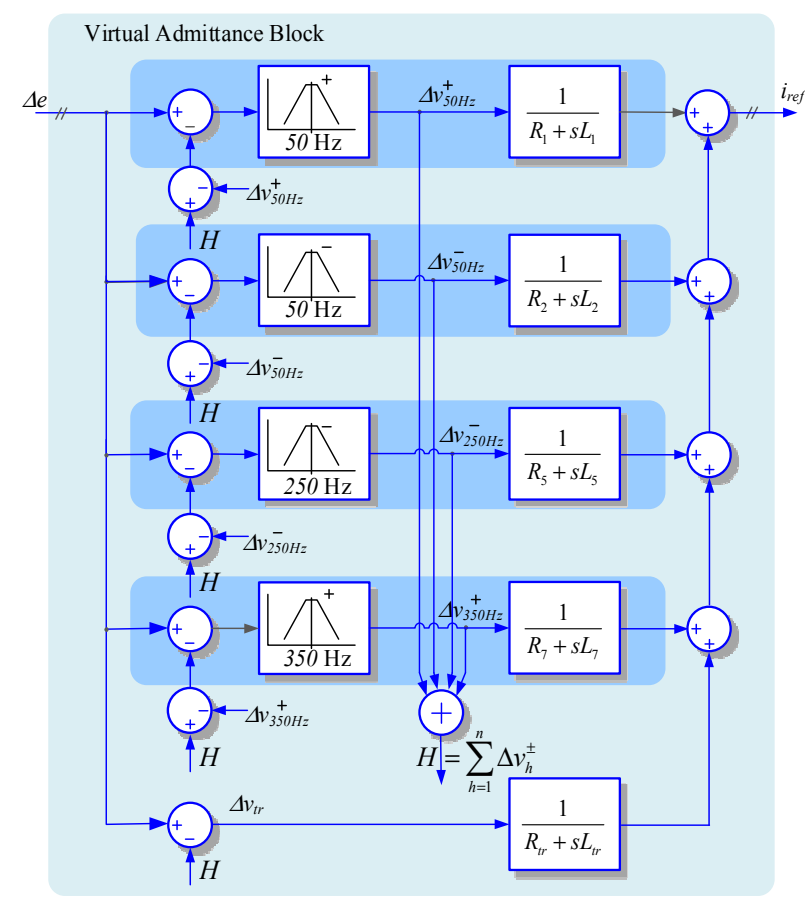

Fig. 2 Virtual admittance block, $Y_{V}(s)$, description for sequence control at different frequencies

The transient branch is the one providing the reference during transient steps, acting as a high pass filter on the reference, while the positive sequence controller is the predominant at the $50 \mathrm{~Hz}$ on the steady state. Assuming that further harmonics are detected, the harmonic compensation loop will generate a current reference in order to remove the voltage distortion at the PCC. On the next sections simulation and experimental results are going to be presented. First validating the control algorithm on the simulation scenario and then demonstrating its capability to restore the voltage at the PCC on the experimental setup.

\section{SimULATION RESUlTS}

The simulation setup is presented in Fig. 3. It consists on an inverter connected to the grid and a harmonic load. This harmonic load is going to absorb harmonic current, adding harmonic perturbation to the grid voltage at the inverter connection point.

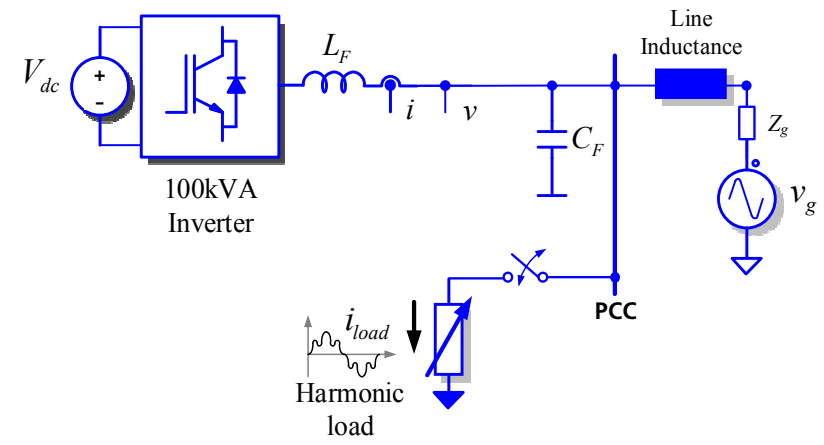

Fig. 3: Simulation setup. $100 \mathrm{kVA}$ SPC based inverter and a harmonic load
For different grid voltage harmonic composition, the control algorithm based on several virtual admittances on parallel is able to generate a current reference to attenuate the harmonic effect on the grid voltage at the PCC. In order to check the correct performance of the controller the test consists of two main stages. The first one considers just one harmonic perturbation affecting the system. The second stage considers a higher harmonic perturbation with several harmonics injection to grid.

The simulations results are showed below on Fig. 4, where two different attenuations of the grid harmonic distortion are achieved. Case a) presents and attenuation of a $10 \%$ voltage distortion of the $5^{\text {th }}$ harmonic. The inverter is capable of seeing the voltage perturbation due to the harmonic load, and injects a specific harmonic current in order to maintain the sinusoidal shape on the voltage at the PCC. In case b) an attenuation of a $10 \%$ voltage distortion of a $7^{\text {th }}$ harmonic is achieved. Including on both cases the virtual admittance block control it is possible to attenuate different harmonics appearing at the grid.
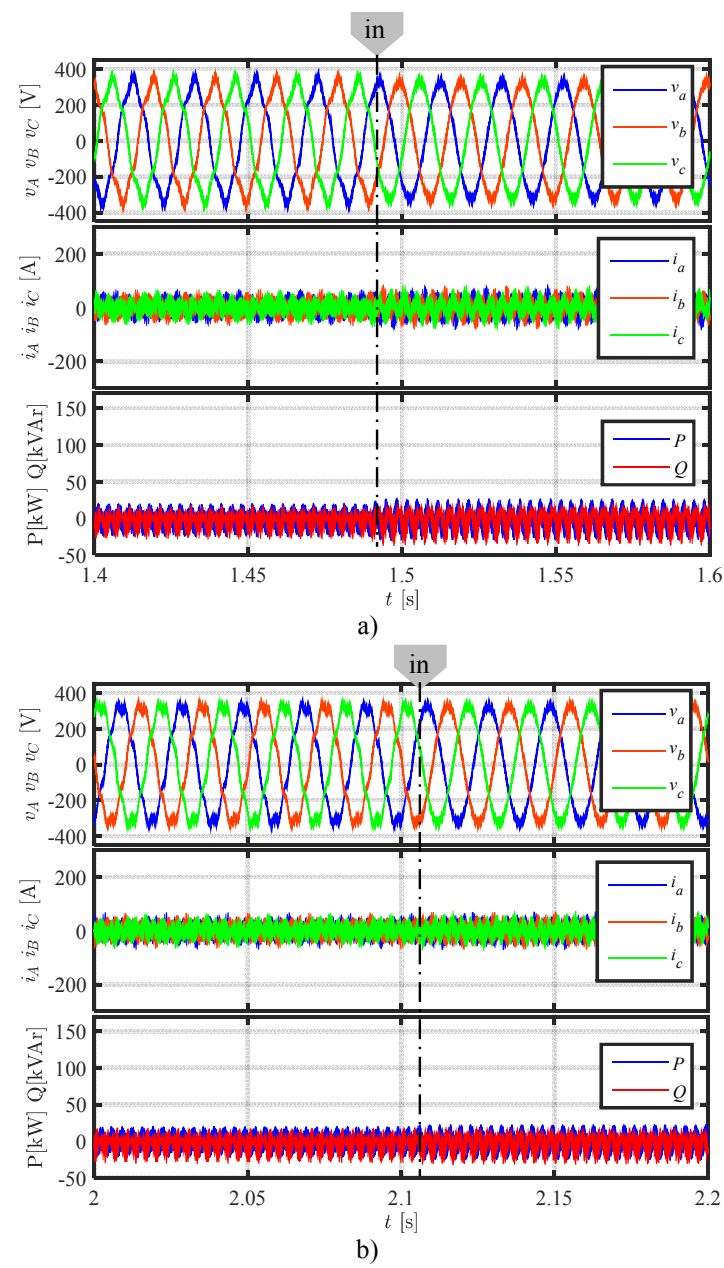

Fig. 4: Simulations results with different harmonic distortions. a) negative sequence $5^{\text {th }}$ harmonic compensation at $t=1.49 \mathrm{~s}$. b) positive sequence $7^{\text {th }}$ harmonic compensation at $\mathrm{t}=2.1 \mathrm{~s}$. 
No matter the harmonic profile, the virtual admittance is capable of detecting the value of the harmonic distortion, its phase, and inject current to attenuate the harmonic distortion on the inverter output voltage.

A second simulation test is being achieved on Fig. 5 where the capability of the SPC to compensate the grid voltage harmonic individually is tested. A programmable load consuming the $5^{\text {th }}$ and $7^{\text {th }}$ harmonic is connected to the PCC. First on Fig. 5a the $5^{\text {th }}$ harmonic compensation is activated at $t=1.77 \mathrm{~s}$ to the control algorithm, and when this happens the $5^{\text {th }}$ harmonic perturbation on the voltage is reduced to a certain value. Then in $\mathrm{t}=2.76 \mathrm{~s}$ the $7^{\text {th }}$ harmonic compensation branch is activated on Fig. 5b. The overall response of the system is to attenuate the specific harmonics determined on the virtual admittances control loop.

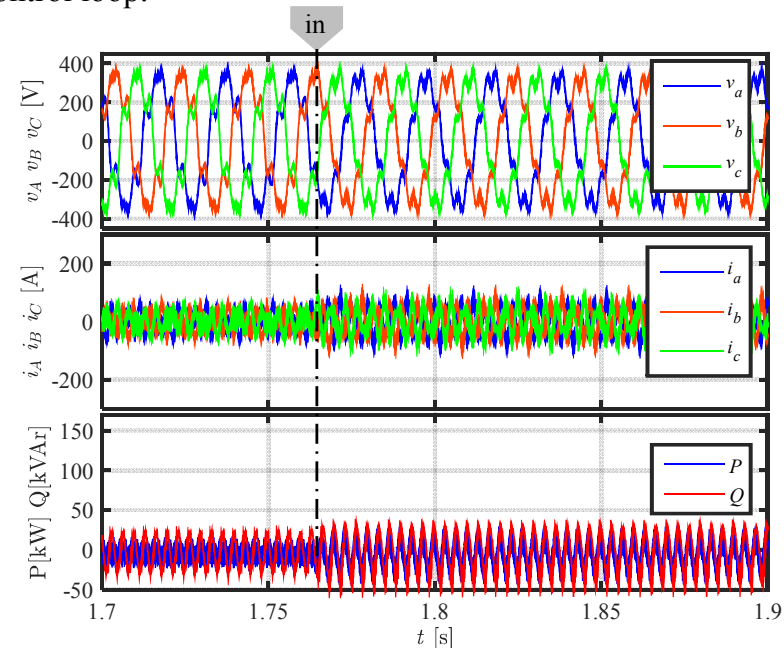

a)

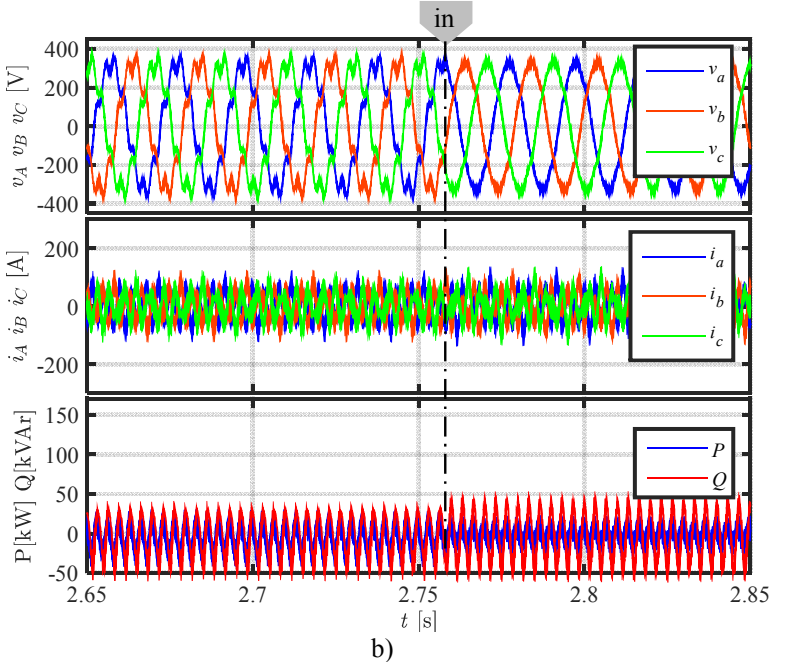

Fig. 5: Simulation results with different harmonics distortions. Activation of the $5^{\text {th }}$ and $7^{\text {th }}$ virtual admittance control to attenuate the harmonic perturbation on grid voltage. a) Activation of the first stage of attenuations $5^{\text {th }}$ virtual admittance b) Activation of the second stage of attenuation $7^{\text {th }}$ virtual admittance.

\section{EXPERIMENTAL RESULTS}

The schematic of the experimental setup is presented in Fig. 3. As it is depicted in the figure it consists on a $100 \mathrm{kVA} \mathrm{SPC}$ based power converter connected to grid, which includes an additional inductance to increase the voltage perturbation in front of harmonics.
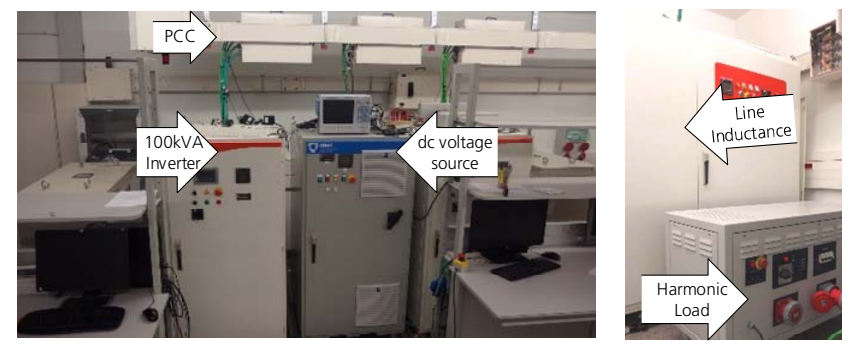

Fig. 6: Experimental setup. 100kVA SPC based inverter and a harmonic load.

On the first test the converter is connected both to the grid and to a harmonic load. This harmonic load absorbs harmonic currents, which give rise to voltage distortions at the PCC due to the effect of the line inductance.

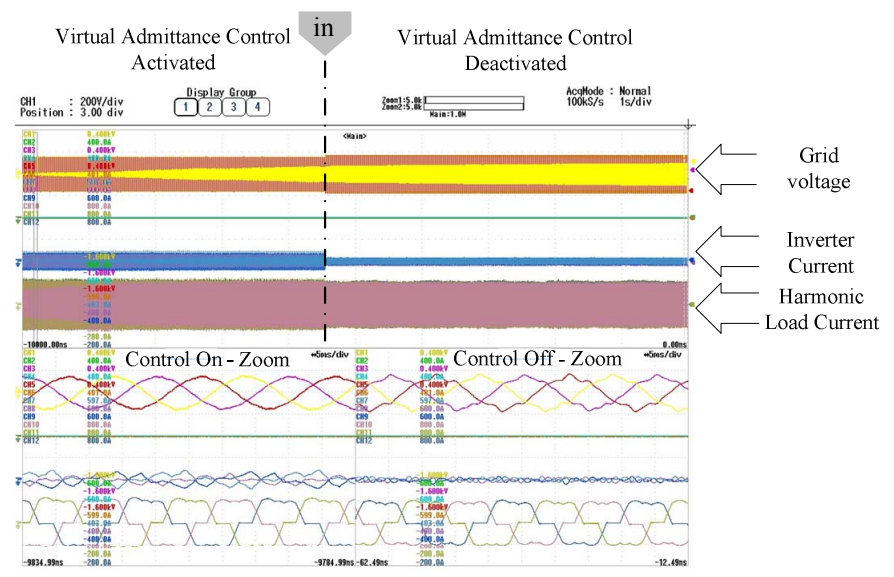

Fig. 7: Power converter grid harmonic compensation capability over a harmonic load perturbation

In this first test, the main goal to achieve with the proposed strategy is to compensate the voltage distortion produced by the harmonic load, by means of injecting the appropriate harmonic current. In Fig. 7 both situations, with and without compensation, can be seen. At the right side, the harmonic load is absorbing harmonics from the grid and it's producing visible voltage perturbations at the PCC. On this side of the figure, there is no compensation by the control showed above on Fig. 2. At the left side of the figure, the harmonic compensation is enabled. It can be seen that if the control algorithm based on virtual admittances at different frequencies is activated, the voltage at the PCC point is clearly improved. By adding the voltage error into the control, the virtual admittance is capable of generating such a current reference that attenuates the effects produced by the harmonic load.

If the FFT analysis is applied in both scenarios, a remarkable difference between voltage harmonics at the point of 
connection of the converter can be seen. In Fig. 8, the FFT of both parts of the signal has been performed. On Fig. 8a the distorted voltage is generated by the harmonic load, and it has in overall a voltage THD of $9.05 \%$. By adding the control algorithm based on the virtual admittance control, on Fig. $8 \mathrm{~b}$ it is possible to see how the attenuation of the harmonic components is achieved. In this study case is reduced to a THD of $3.73 \%$.
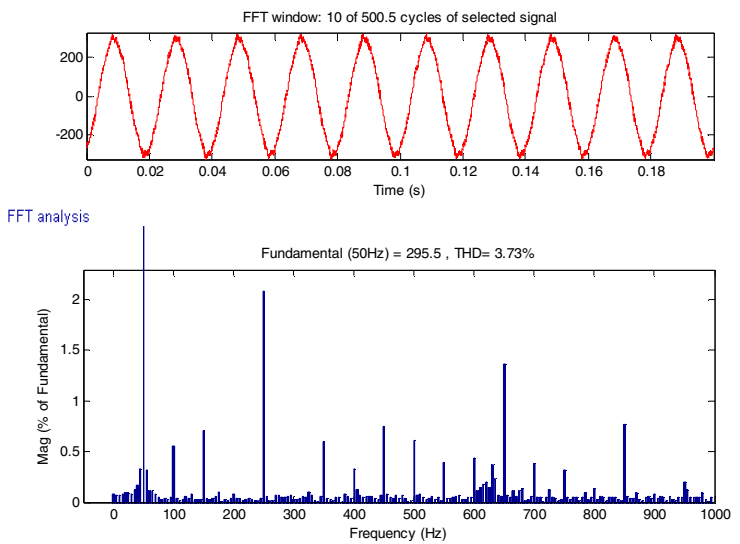

a)
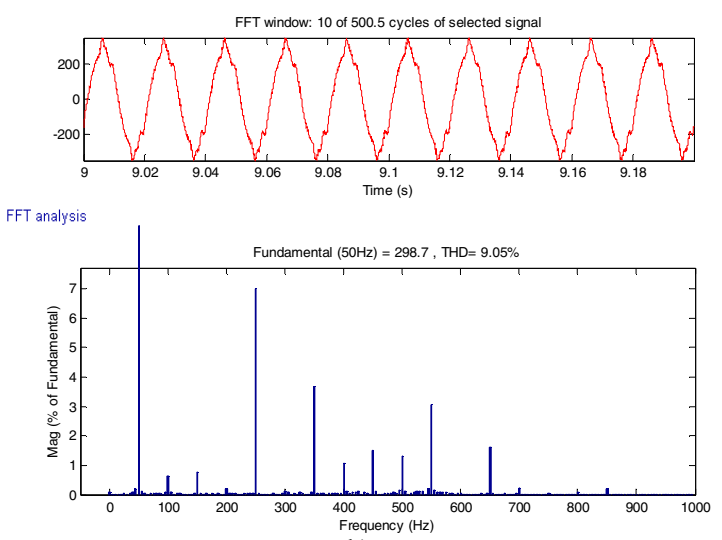

b)

Fig. 8. FFT Analysis of $v_{\mathrm{PCC}}$ on the left side zoom and the right side zoom respectively

\section{CONCLUSIONS}

In this paper an extended version of the SPC controller, devoted to enhance the voltage profile at the PCC of the converter due to harmonic distortions, has been presented. The control structure has been shown, which finally results in a feasible and effective algorithm able to counteract the voltage distortion. The simulation results have proven the good performance of the proposal considering different scenarios. Likewise, the experimental results have also shown the voltage distortion reduction achieved in a real scenario.

Different techniques can be used for harmonic reduction at the PCC, which involves the installation of passive or active compensators. Those elements can affect the stability during transients and island mode. This prompts the system to include a selective harmonic compensation into the power converter control, which will be able to improve the voltage distortion at the PCC and share the harmonic current that is being supplied by the grid to the local harmonic load.

On this paper an effective solution to voltage harmonic compensation at the PCC is exposed and verified by means of simulations and experimental results. Where each power converter unit is able to, by means of a virtual admittance tuned for specific harmonic value, compensate the voltage distortion generated by a harmonic load through injecting the specific harmonic into the grid. Thus reducing the absorption of harmonic current from the grid and being supplied directly from the converter harmonic current.

\section{ACKNOWLEDGMENT}

This work has been partially supported by the Spanish Ministry of Economy and Competitiveness under the Project ENE201460228-R and ENE2013-48428-C2-2-R

P. Rodríguez, J.-I. Candela, J. Rocabert, and R. Teodorescu, "Synchronous power controller for a generating system based on static power converters," WO 2012117131 A1, 2012.

[2] Q.-C. Zhong and G. Weiss, "Synchronverters: Inverters That Mimic Synchronous Generators," IEEE Trans. Ind. Electron., vol. 58, no. 4, pp. 1259-1267, Apr. 2011.

[3] Qing-Chang Zhong, Phi-Long Nguyen, Zhenyu Ma, and Wanxing Sheng, "Self-Synchronized Synchronverters: Inverters Without a Dedicated Synchronization Unit," IEEE Trans. Power Electron., vol. 29, no. 2, pp. 617-630, Feb. 2014.

[4] P. Rodriguez, J.-I. Candela, J. Rocabert, and R. Teodorescu, "Virtual controller of electromechanical characteristics for static power converters," WO2012117132A1, 2012.

[5] W. Zhang, A. M. Cantarellas, J. Rocabert, A. Luna, and P. Rodriguez, "Synchronous Power Controller With Flexible Droop Characteristics for Renewable Power Generation Systems," IEEE Trans. Sustain. Energy, vol. 7, no. 4, pp. 1572-1582, Oct. 2016.

[6] P. Rodriguez, J.-I. Candela, J. Rocabert, and R. Teodorescu, "Virtual admittance controller based on static power converters," WO 2012117133 A1, 2012.

[7] K. De Brabandere, B. Bolsens, J. Van den Keybus, A. Woyte, J. Driesen, and R. Belmans, "A Voltage and Frequency Droop Control Method for Parallel Inverters," IEEE Trans. Power Electron., vol. 22, no. 4, pp. 1107-1115, Jul. 2007.

[8] T. Loix, K. De Brabandere, J. Driesen, and R. Belmans, "A Three-Phase Voltage and Frequency Droop Control Scheme for Parallel Inverters," in IECON 2007 - 33rd Annual Conference of the IEEE Industrial Electronics Society, 2007, pp. $1662-1667$.

[9] L. Zhang, L. Harnefors, and H.-P. Nee, "PowerSynchronization Control of Grid-Connected Voltage-Source Converters," IEEE Trans. Power Syst., vol. 25, no. 2, pp. 809-820, May 2010. 